\title{
On the Solution of the Equations Arising from Collocation with Cubic $B$-Splines
}

\author{
By Richard F. Sincovec
}

\begin{abstract}
An iterative technique for solving the equations arising from collocation with cubic $B$-splines in solving second-order nonlinear boundary-value problems is defined and shown to converge.
\end{abstract}

1. Introduction. Consider the boundary-value problem of the form

$$
\begin{gathered}
y^{\prime \prime}=f\left(x, y, y^{\prime}\right), \quad 0<x<1, \\
y(0)=A, \quad y(1)=B .
\end{gathered}
$$

We assume that $f(x, y, z)$ has continuous derivatives which satisfy

$$
|\partial f / \partial z| \leqq P^{*}, \quad 0<Q_{*} \leqq \partial f / \partial y \leqq Q^{*},
$$

for some positive constants $P^{*}, Q^{*}$, and $Q_{*}$. The assumptions (1.2) not only guarantee the existence and uniqueness of a solution of (1.1) but are essential for the development of the theorem that is given in Section 3.

In this note, we consider the solution of the sets of equations that arise when problem (1.1) is solved by the usual collocation method using cubic $B$-splines, [1], [3] and [5]. In particular, we give an explicit condition on the mesh size based on (1.2) such that all approximating problems with sufficiently fine mesh have unique solutions which can be obtained by an explicit, globally convergent iteration.

2. Derivation of Equations. Let $\pi$ be a uniform partition of $[0,1]: 0=x_{0}<$ $x_{1}<\cdots<x_{N}=1$, let $h$ denote the partition width $1 / N$, and let $\operatorname{Sp}^{(3)}(\pi)$ be a spline function of third degree subordinate to $\pi$. The space $\operatorname{Sp}^{(3)}(\pi)$ has dimension $N+3$. A basis for $\mathrm{Sp}^{(3)}(\pi)$ consisting of splines of minimal support, which we denote by $S_{i}, i=-1,0,1, \cdots, N+1$, is given by Schoenberg [5]. The following table of values will be all we need:

\begin{tabular}{cccccc} 
& $x_{i-2}$ & $x_{i-1}$ & $x_{i}$ & $x_{i+1}$ & $x_{i+2}$ \\
\cline { 2 - 6 }$S_{i}$ & 0 & $1 / 4$ & 1 & $1 / 4$ & 0 \\
$S_{i}^{\prime}$ & 0 & $3 / 4 h$ & 0 & $-3 / 4 h$ & 0 \\
$S_{i}^{\prime \prime}$ & 0 & $3 / 2 h^{2}$ & $-3 / h^{2}$ & $3 / 2 h^{2}$ & 0
\end{tabular}

Received August 31, 1971, revised March 6, 1972.

AMS 1970 subject classifications. Primary 65N35, 65L10.

Key words and phrases. Boundary-value problems, collocation, spline functions.

Copyright $@ 1972$, American Mathematical Society 
We seek an approximate solution of (1.1) in the form of a cubic spline subordinate to $\pi$ :

$$
S=\sum_{i=-1}^{N+1} \alpha_{i} S_{i}
$$

by forcing it to satisfy the boundary condition as well as the differential equation exactly at $N+1$ points in $[0,1]$. It is natural, though it seems by no means necessary, to require the satisfaction of the differential equation precisely at the joints of $\pi$. Thus, we have the following system to solve for $\alpha=\left(\alpha_{i}\right)$ :

$$
\begin{gathered}
\sum_{i=-1}^{N+1} \alpha_{i} S_{i}^{\prime \prime}\left(x_{i}\right)=f\left(x, S\left(x_{i}\right), S^{\prime}\left(x_{i}\right)\right), \quad i=0,1, \cdots, N, \\
\sum_{i=-1}^{N+1} \alpha_{j} S_{j}(0)=A, \quad \sum_{i=-1}^{N+1} \alpha_{j} S_{i}(1)=B .
\end{gathered}
$$

The existence, uniqueness, and convergence rates for spline approximations to (1.1) via collocation have been established in very general circumstances, [1], [3], and [4], which, of course, is a primary virtue of collocation. The difficulty is in giving circumstances in which one can guarantee that he can solve the approximating problem (2.3) by an explicitly defined method.

3. Solution of Equations. The Eqs. (2.3a) are, from Table (2.1),

$$
\alpha_{i-1}-2 \alpha_{i}+\alpha_{i+1}=\frac{2}{3} h^{2} f\left(x_{i}, S\left(x_{i}\right), S^{\prime}\left(x_{i}\right)\right), \quad i=0,1, \cdots, N,
$$

where $S\left(x_{i}\right)$ is given by $(2.2)$ and $S^{\prime}\left(x_{i}\right)$ is the derivative of $S(x)$ evaluated at $x=x_{i}$. The simplicity in forming (3.1) illustrates another primary virtue of collocation. That is, forming the approximating problem via collocation is nearly as easy as traditional finite differences in contrast to Galerkin procedures.

We propose the following iteration scheme for solving this set of nonlinear equations:

$$
\begin{aligned}
& \alpha_{i}^{0}=\text { arbitrary, } \quad 0 \leqq i \leqq N, \\
& \alpha_{i}^{\nu+1}=(1+\omega)^{-1}\left[\frac{1}{2}\left(\alpha_{i+1}^{\nu}+\alpha_{i-1}^{\nu}\right)+\omega \alpha_{i}^{\nu}-\frac{h^{2}}{3} f\left(x_{i}, S\left(x_{i}, \alpha^{\nu}\right), S^{\prime}\left(x_{i}, \alpha^{\nu}\right)\right)\right], \\
& 0 \leqq i \leqq N, \quad \nu=0,1, \cdots, \\
& \alpha_{-1}^{\nu}=4 A-4 \alpha_{0}^{\nu}-\alpha_{1}^{\nu}, \quad \alpha_{N+1}^{\nu}=4 B-4 \alpha_{N}^{\nu}-\alpha_{N-1}^{\nu}, \quad \nu=0,1, \cdots,
\end{aligned}
$$

where we write $S(x, \alpha)$ to indicate which values of $\alpha$ are used in (2.2). Equations (3.2c) are obtained from Eqs. (2.3b) with the aid of Table (2.1).

We can now state the following:

THEOREM. Let $f(x, y, z)$ have continuous derivatives which satisfy (1.2). Then the system of equations (2.3) has a unique solution for each $h$ such that $h^{2} Q^{*} / 3+$ $h P^{*} \leqq 2$. This solution is the limit of the iterates $\left\{\alpha_{i}^{(\nu)}\right\}$ as $\nu \rightarrow \infty$ defined by (3.2) with any finite $\omega$ satisfying $\omega \geqq h^{2} Q^{*} / 3$. The convergence factor for this scheme is at most

$$
\lambda(\omega) \equiv 1-h^{2} Q_{*} / 2(1+\omega) .
$$


The proof of the preceding theorem is essentially identical to that given in [2] for the finite-difference treatment of problem (1.1) and hence it will not be given here.

An important observation is that the scheme (3.2) converges for any initial estimate $\alpha_{i}^{(0)}, 0 \leqq j \leqq N$, provided $\omega \geqq h^{2} Q^{*} / 3$. The analysis also reveals that the best value for $\omega$ is the limiting value $\omega=h^{2} Q^{*} / 3$. Of course, even with this choice, the convergence may be extremely slow since $\lambda(\omega)=1-O\left(h^{2}\right)$ can be close to unity.

The extension of these results to the more general boundary conditions

$$
g_{1}\left(y(0), y^{\prime}(1)\right)=0, \quad g_{2}\left(y(1), y^{\prime}(1)\right)=0,
$$

creates no essential difficulties if (3.3) can be used to obtain successive approximations to $\alpha_{-1}$ and $\alpha_{N+1}$ as well as preserve the contraction property of the iterative scheme.

As a final remark, it should be noted that the proof of the Theorem yields an elementary proof of convergence of cubic spline collocation approximations to the solution of the nonlinear boundary-value problem.

Computer Science Department

Kansas State University

Manhattan, Kansas 66502

1. C. DE Boor, The Method of Projections as Applied to the Numerical Solution of Two Point Boundary Value Problems Using Cubic Splines, Dissertation, University of Michigan, Ann Arbor, Mich., 1966.

2. H. B. Keller, Numerical Methods for Two-Point Boundary-value Problems, Blaisdell, Waltham, Mass., 1968. MR 37 \#6038.

3. T. R. LuCAS \& G. W. ReDDIEN, JR., "Some collocation methods for nonlinear boundary value problems," SIAM J. Numer. Anal., v. 9, 1972, pp. 341-356.

4. R. D. Russell \& L. F. Shampine, "A collocation method for boundary value problems," Numer. Math., v. 19, 1972, pp. 1-28.

5. I. J. Schoenberg, "On spline functions," Inequalities (Proc. Sympos. Wright-Patterson Air Force Base, Ohio, 1965), Academic Press, New York, 1967, pp. 255-291. MR 36 \#6848. 